\title{
Factores de riesgo cardiovascular asociados a ectasia coronaria e infarto agudo de miocardio
}

\author{
Eduardo Rojas-Milán', Cristina E. Morales de León², Andrés García-Rincón, Ma. Pilar Cruz-Domínguez, \\ Irvin Ordoñez-González ${ }^{5}$, Rommy del C. Santos-Sánchez ${ }^{6}$, Joel E. Morales-Gutiérrez ${ }^{6}$, Olga Vera-Lastra ${ }^{1}$ \\ y Gabriela Medina ${ }^{7 *}$ \\ ${ }^{1}$ Departamento de Medicina Interna, Hospital de Especialidades Centro Médico Nacional La Raza, Ciudad de México, Méx.; ${ }^{2}$ Unidad Coronaria, \\ Hospital de Especialidades Centro Médico Nacional La Raza, Ciudad de México, Méx.; ${ }^{3}$ Departamento de Hemodinamia, Hospital de Especialidades \\ Centro Médico Nacional La Raza, Ciudad de México, Méx.; ${ }^{4}$ División de Investigación en Salud, Hospital de Especialidades Centro Médico Nacional \\ La Raza, Ciudad de México, Méx.; ${ }^{5}$ Facultad de Medicina, Universidad Veracruzana, Campus Veracruz, Ver.; ${ }^{6}$ Escuela de Medicina, División \\ Académica de Ciencias de la Salud, Universidad Juárez Autónoma de Tabasco, Tab.; ${ }^{\top}$ Unidad de Investigación en Medicina Traslacional en \\ enfermedades hemato-oncológicas, Hospital de Especialidades Centro Médico Nacional La Raza, Ciudad de México, Méx. México
}

\section{Resumen}

Introducción: La ectasia coronaria tiene baja prevalencia en población general, los factores de riesgo pueden diferir de la enfermedad arterial coronaria. Objetivo: Identificar la prevalencia de ectasia coronaria en pacientes con infarto agudo de miocardio (IAM) y factores de riesgo cardiovascular (FRCV). Métodos: Estudio retrospectivo, transversal. De 3,254 cateterismos cardiacos por IAM durante un año, 2,975 no presentaron ectasia coronaria. Se incluyeron 558 pacientes clasificados como portadores de ectasia coronaria en coronariografía y controles aquellos con características similares exceptuando la ectasia coronaria y se registraron los FRCV. Empleamos estadística descriptiva, análisis bivariante, multivariante y calculamos el odds ratio (OR). Resultados: Se estudiaron 279 pacientes con y sin ectasia coronaria. La prevalencia de ectasia coronaria fue del 8.5\%. El índice plaqueta/linfocito (IPL) se encontró más elevado en pacientes con ectasia que en aquellos sin ectasia $(p=0.003)$. En el análisis bivariante los FRCV asociados fueron sobrepeso, obesidad y diabetes, $y$ en el multivariante la hipercolesterolemia (OR: 3.90; $p=0.0001$ ) y exposición a herbicidas (OR: 6.82; $p=0.020$ ). Conclusiones: Encontramos alta prevalencia de ectasia coronaria, los principales factores de riesgo fueron el antecedente de uso de herbicidas e hipercolesterolemia. Identificamos el IPL elevado en estos pacientes. Es importante la detección oportuna debido a su asociación con eventos coronarios agudos.

PALABRAS CLAVE: Enfermedad coronaria. Infarto de miocardio. Prevalencia. Herbicidas. Angiografía.

\section{Cardiovascular risk factors associated with coronary ectasia and acute myocardial infarction}

\section{Abstract}

Introduction: Coronary ectasia has a low prevalence in the general population. Its risk factors may differ from those of coronary artery disease. Objective: To identify the prevalence of coronary ectasia in patients with acute myocardial infarction (AMI) and cardiovascular risk factors (CVRFs). Methods: Retrospective, cross-sectional study. Out of 3,254 cardiac catheterizations for AMI during one year, 2,975 had no coronary ectasia. We included 558 patients with coronary ectasia on coronary angiography and, as controls, subjects with similar characteristics except for coronary ectasia, and CVRFs were recorded. Descriptive

Correspondencia:

*Gabriela Medina

E-mail: dragabymedina@yahoo.com.mx
Gac Med Mex. 2021;157:623-629

Disponible en PubMed

www.gacetamedicademexico.com

0016-3813/C 2021 Academia Nacional de Medicina de México, A.C. Publicado por Permanyer. Este es un artículo open access bajo la licencia CC BY-NC-ND (http://creativecommons.org/licenses/by-nc-nd/4.0/). 
statistics, bivariate and multivariate analysis were used; odds ratio (OR) was calculated. Results: 279 patients with and without coronary ectasia were studied. The prevalence of coronary ectasia was $8.5 \%$. The platelet/lymphocyte ratio (PLR) was higher in patients with ectasia than in those without ectasia $(p=0.003)$. In the bivariate analysis, associated CVRFs were overweight, obesity and diabetes, and in the multivariate analysis, hypercholesterolemia (OR: 3.90; $p=0.0001)$ and exposure to herbicides (OR: 6.82; $p=0.020$ ). Conclusions: $A$ high prevalence of coronary ectasia was found, with the main risk factors being a history of herbicide use and hypercholesterolemia. PLR was found to be elevated in these patients. Early detection is important due to its association with acute coronary events.

KEY WORDS: Coronary artery disease. Myocardial infarction. Prevalence. Herbicides. Angiography.

\section{Introducción}

El término ectasia coronaria, acuñado en 1966 se define como la dilatación coronaria que excede el diámetro normal de los segmentos adyacentes o el diámetro de la arteria coronaria más grande por lo menos 1.5 veces $^{1,2}$.

La aterosclerosis es su principal causa, con predominio en el sexo masculino y asociación con factores de riesgo cardiovascular (FRCV) tradicionales, correspondiendo a más del $50 \%$ de los casos. El $30 \%$ es causado por anomalías congénitas y el $20 \%$ restante por etiologías autoinmunes, enfermedad del tejido conectivo, causas infecciosas y postraumáticas, así como el uso de herbicidas y consumo de cocaína. La arteria coronaria derecha es la más afectada (40-61\%), seguido de la descendente anterior (15-32\%) y circunfleja izquierda (15-23\%). El 75\% de las ectasias son de vaso único. Más del $80 \%$ de las ectasias se asocian a enfermedad arterial coronaria obstructiva ${ }^{3-5}$.

El estudio más grande fue realizado en China, donde de 42,800 cateterismos cardiacos se reportó una prevalencia de ectasia coronaria del $1.3 \%$ asociada al sexo masculino, hipertensión arterial sistémica, tabaquismo, dislipidemia y solamente el $27.5 \%$ contaba con infarto de miocardio. En países asiáticos como Singapur, India, Irán y Turquía se documentaron prevalencias de 1.2, 1.9, 2.3 y $4.2 \%$, con antecedente de infarto de miocardio en el $13-25 \%$ de su población ${ }^{6-9}$.

En España se reportó una prevalencia de ectasia coronaria del $3.4 \%$ en un total de 4,332 coronariografías analizadas, de las cuales el $40.8 \%$ manifestó infarto de miocardio ${ }^{10}$. La prevalencia más baja a nivel mundial $(0.4 \%)$ se observó en un estudio sueco-irlandés, en donde de 16,454 angiografías, el 62\% contaba con antecedente de enfermedad isquémica cardiaca $^{11}$. La cifra más alta $(6 \%)$ fue documentada en Arabia Saudita, en donde se analizaron 1,115 cateterismos $^{12}$.
En México solo existen dos publicaciones sobre el tema donde se analizaron cateterismos en hospitales de tercer nivel, reportando prevalencias del 2.3 y $4.5 \%$, de los cuales el 22 y $84.6 \%$ presentaron infarto de miocardio, respectivamente ${ }^{13,14}$. En ambos estudios no hubo asociación clara de los FRCV en relación con la de otros países. El objetivo del estudio fue investigar la prevalencia de la ectasia coronaria en pacientes con infarto agudo de miocardio y analizar los FRCV asociados a esta.

\section{Métodos}

El presente estudio se efectuó en los servicios de hemodinamia y unidad coronaria de un hospital de tercer nivel en la Ciudad de México. El diseño del estudio fue observacional, retrospectivo, de casos y controles.

Para los casos se incluyeron pacientes portadores de infarto agudo de miocardio, de ambos sexos, mayores de 18 años de edad, con hallazgo concomitante de ectasia coronaria en coronariografía. No se incluyeron pacientes con diagnóstico al egreso de angina inestable o de angina crónica estable. Se excluyeron los pacientes en quienes no se obtuvo el $80 \%$ de la información requerida para el análisis.

Para el grupo control se incluyeron pacientes con diagnóstico de infarto agudo de miocardio sin ectasia coronaria en coronariografía, de ambos sexos, mayores de 18 años de edad, con criterios de no inclusión y exclusión similares a los casos. Por cada paciente con ectasia coronaria se incluyó un control en relación 1:1. Los casos del grupo control se obtuvieron en forma aleatoria del total de procedimientos de coronariografía efectuados en ese periodo.

\section{Metodología}

El proyecto fue aprobado por el comité local de investigación, con número de registro R2018-3501116. Los expedientes revisados fueron seleccionados 
Tabla 1. Factores de riesgo cardiovasculares en pacientes con y sin ectasia coronaria

\begin{tabular}{|c|c|c|c|}
\hline Variable & $\begin{array}{l}\text { Infarto agudo de miocardio sin ectasia } \\
\qquad(\mathrm{n}=279)\end{array}$ & $\begin{array}{l}\text { Infarto agudo de miocardio con ectasia } \\
\qquad(\mathrm{n}=279)\end{array}$ & p \\
\hline Sexo femenino & $68(24.4 \%)$ & $35(12.5 \%)$ & $0.001^{*+}$ \\
\hline Sexo masculino & $211(75.6 \%)$ & $244(87.5 \%)$ & $0.001^{*+}$ \\
\hline Diabetes mellitus & $141(50.5 \%)$ & $102(36.6 \%)$ & $0.001^{*+}$ \\
\hline Hipertensión arterial & $193(69.2 \%)$ & $183(65.6 \%)$ & $0.367^{\star}$ \\
\hline Tabaquismo & $158(56.6 \%)$ & $158(56.6 \%)$ & $1^{*}$ \\
\hline Hipercolesterolemia & $64(23.1 \%)$ & $132(47.3 \%)$ & $0.001^{*+}$ \\
\hline Uso de cocaína & $1(0.4 \%)$ & $1(0.4 \%)$ & $1^{*}$ \\
\hline Exposición a herbicidas & $2(0.7 \%)$ & $12(4.3 \%)$ & $0.007^{*+}$ \\
\hline Enfermedades autoinmunes & $5(1.8 \%)$ & $3(1.1 \%)$ & $0.476^{*}$ \\
\hline Obesidad & $55(19.7 \%)$ & $126(45.2 \%)$ & $0.001^{*+}$ \\
\hline Sobrepeso & $117(42.2 \%)$ & $161(57.8 \%)$ & $0.001^{*+}$ \\
\hline
\end{tabular}

de la base de datos y expedientes electrónicos del servicio de unidad coronaria, de los pacientes que estuvieron hospitalizados en dichos servicios en el lapso de julio 2018 a julio 2019 y a los cuales se les realizó coronariografía. Se obtuvieron datos por cada paciente que incluyeron: nombre, edad, sexo, FRCV tradicionales (tabaquismo, obesidad, sobrepeso, antecedente de hipercolesterolemia e hipertrigliceridemia, diabetes mellitus, hipertensión arterial sistémica) de acuerdo con la historia clínica, FRCV no tradicionales (uso de cocaína, enfermedades autoinmunes, enfermedades del tejido conectivo como síndrome de Marfan o síndrome de Elhers Danlos, exposición a herbicidas), así como parámetros de laboratorio al ingreso a unidad coronaria tales como cuenta total de linfocitos, hemoglobina, ácido úrico, leucocitos, polimorfonucleares, plaquetas, índice neutrófilo: linfocito, índice plaqueta: linfocito (IPL), ancho de distribución eritrocitaria, creatina fosfocinasa, creatina fosfocinasa fracción MB, albúmina, colesterol total, colesterol vinculado a lipoproteínas de baja densidad (C-LDL) y triglicéridos. Todos estos parámetros registrados al momento de su ingreso a unidad coronaria.

\section{Análisis estadístico}

Se empleó estadística descriptiva con medidas de tendencia central (media, mediana), desviación estándar, rangos, porcentajes; para la comparación de variables sin distribución normal se utilizó la prueba U de Mann-Whitney. Se efectuó análisis de regresión logística bivariante para medir el efecto de cada factor sobre la ectasia coronaria y uno multivariante con razón de momios (RM) para evaluar qué variable podía predecir como factor de riesgo independiente de esta entidad y se estableció un valor de $p<0.05$ como estadísticamente significativo. El análisis estadístico se efectuó mediante el programa SPSS versión 25.0 (Chicago, Illinois), desarrollado por International Business Machines Corporation (IBM).

\section{Resultados}

Analizamos un total de 558 procedimientos de cateterismo cardiaco por infarto agudo de miocardio durante el lapso de un año. De ellos, 279 pacientes con infarto sin ectasia coronaria y 279 con ectasia coronaria. Del grupo con ectasia coronaria, la relación hombre: mujer fue 2.5:1, además el 57.8\% tenían sobrepeso, el $45.2 \%$ obesidad, el $36.6 \%$ fueron portadores de diabetes mellitus y el $4.3 \%$ tuvieron antecedente de exposición a herbicidas (Tabla 1).

De los 279 con ectasia coronaria el 49.1\% presentó ectasia Markis I (afección de dos o más vasos), el $13.97 \%$ ectasia Markis II (afección de un vaso y un segmento de otro), el 27.95\% ectasia Markis III (afección de un solo vaso) y el $8.96 \%$ ectasia Markis IV (afección en un segmento de un solo vaso). 
Tabla 2. Valores demográficos y paraclínicos en pacientes con y sin ectasia coronaria

\begin{tabular}{|c|c|c|c|}
\hline Variable & Infarto sin ectasia $(n=279)$ & Infarto con ectasia $(n=279)$ & p \\
\hline Edad (años) & $65(56-71)$ & $63(56-71)$ & $0.325^{\star}$ \\
\hline Índice de masa corporal & $24.6(23-27.4)$ & $28(25.8-33.2)$ & $0.0001^{*}$ \\
\hline Linfocitos $\mu / L$ & 1,368 (990-1,782) & $1,376(960-1,859)$ & $0.959^{*}$ \\
\hline Hemoglobina (g/dL) & $14.1(12.7-15.3)$ & $15.2(13.9-16.4)$ & $0.0001^{*}$ \\
\hline Triglicéridos (mg/dL) & $119(88.25-158)$ & $130(99.5-178.5)$ & $0.006^{*+}$ \\
\hline Ácido úrico (mg/dL) & $5.75(4.30-7.05)$ & $5.70(4.80-7.50)$ & $0.604^{*}$ \\
\hline Leucocitos $(\mu \mathrm{L})$ & $9,700(7,300-12,200)$ & $9,300(7,400-12,100)$ & $0.641^{*}$ \\
\hline Polimorfonucleares $(\mu \mathrm{L})$ & $7,128(4,836-9,782)$ & $6,720(4,898-9,625)$ & $0.416^{*}$ \\
\hline Plaquetas $(\mu \mathrm{L})$ & $252,000(200,500-300,200)$ & $217,000(170,500-266,250)$ & $0.000^{*+}$ \\
\hline INL & $4.94(0.46-36.59)$ & $5.07(0.21-64)$ & $0.610^{*}$ \\
\hline $\mathrm{IPL}$ & $160.16(118.25-227.22)$ & $177.79(129.90-252.87)$ & $0.003^{*+}$ \\
\hline ADE & $13.9(13.4-14.4)$ & $13.6(13.2-14.2)$ & $0.001^{\star+}$ \\
\hline $\mathrm{CK}(\mathrm{U} / \mathrm{L})$ & $2,523(1,876-3,621)$ & $2,450(1,836-3,557)$ & $0.832^{*}$ \\
\hline CKMB (U/L) & $452(390-543)$ & $457(391-621)$ & $0.017^{\star+}$ \\
\hline $\mathrm{c}-\mathrm{LDL}(\mathrm{mg} / \mathrm{dl})$ & $104.4(47-171)$ & $88(75.6-124.750)$ & $1^{*}$ \\
\hline Colesterol (mg/dl) & $131(108-168.5)$ & $148(119-180.5)$ & $0.005^{\star+}$ \\
\hline Albúmina (g/dL) & $3.5(3.1-3.8)$ & $3.7(3.3-4)$ & $0.005^{*+}$ \\
\hline
\end{tabular}

La mediana de índice de masa corporal fue mayor en el grupo de ectasia, la mediana de plaquetas fue menor. El IPL fue mayor para el grupo con ectasia $(p=0.003)$ (Tabla 2).

En la caracterización angiográfica se vio más afectada la arteria coronaria derecha (35.1\%), seguido de la descendente anterior (29\%); la arteria menos afectada fue la circunfleja (9\%) (Tabla 3). En la figura 1 se ilustra un ejemplo representativo de ectasia con afectación en arteria coronaria derecha.

En la tabla 4 se muestra el análisis bivariante, encontramos que la diabetes mellitus, el sobrepeso y la obesidad fueron factores asociados a ectasia coronaria. En el análisis multivariante, únicamente la hipercolesterolemia y exposición a herbicidas confirieron riesgo de presentar ectasia coronaria.

\section{Discusión}

Encontramos una prevalencia de ectasia coronaria del $8.5 \%$, siendo de las más altas documentadas en
Tabla 3. Vaso culpable

\begin{tabular}{|l|c|c|}
\hline & $\begin{array}{c}\text { Infarto agudo de } \\
\text { miocardio sin ectasia } \\
(\mathbf{n = 2 7 9 )}\end{array}$ & $\begin{array}{c}\text { Infarto agudo de } \\
\text { miocardio con ectasia } \\
(\mathbf{n}=279)\end{array}$ \\
\hline $\begin{array}{l}\text { Coronaria } \\
\text { derecha }\end{array}$ & $44(15.8)$ & $98(35.1)$ \\
\hline $\begin{array}{l}\text { Arteria } \\
\text { circunfleja }\end{array}$ & $11(3.9)$ & $25(9)$ \\
\hline $\begin{array}{l}\text { Descendente } \\
\text { anterior }\end{array}$ & $0(0)$ & $81(29)$ \\
\hline $\begin{array}{l}\text { Enfermedad } \\
\text { trivascular }\end{array}$ & $89(31.9)$ & $36(12.9)$ \\
\hline $\begin{array}{l}\text { Enfermedad } \\
\text { bivascular }\end{array}$ & $74(26.5)$ & $39(14)$ \\
\hline
\end{tabular}

el mundo, en el contexto de pacientes con infarto agudo de miocardio a los que se les efectuó coronariografía. Existió predominio en hombres, un $87.5 \%$, en contraste con el $12.5 \%$ de mujeres con ectasia 
Tabla 4. Variables clínicas en el modelo de regresión logística para ectasia coronaria

\begin{tabular}{|l|c|c|c|c|c|c|}
\hline & \multicolumn{3}{|c|}{ Análisis bivariante } & \multicolumn{3}{c|}{ Análisis multivariante } \\
\cline { 2 - 7 } & Viesgo & IC 95\% & p & OR & IC 95\% para OR \\
\hline Diabetes mellitus & 1.382 & $(1.1-1.6)$ & $0.001^{*}$ & 0.412 & $(0.2-0.6)$ & 0.0001 \\
\hline Sobrepeso & 1.730 & $(1.3-2.2)$ & $0.0001^{*}$ & 0.171 & $(0.10-0.27)$ & 0.0001 \\
\hline Obesidad & 1.983 & $(1.5-2.5)$ & $0.0001^{*}$ & 0.135 & $(0.08-0.21)$ & 0.0001 \\
\hline Hipercolesterolemia & 0.492 & $(0.3-0.6)$ & 0.0001 & 3.90 & $(2.5-5.9)$ & $0.0001^{*}$ \\
\hline Exposición a herbicidas & 0.167 & $(0.03-0.7)$ & 0.012 & 6.82 & $(1.34-34)$ & $0.020^{*}$ \\
\hline Tabaquismo & 1 & $(0.8-1.1)$ & 1 & 1.205 & $(0.8-1.7)$ & 0.352 \\
\hline Hipertensión arterial & 1.055 & $(0.9-1.1)$ & 0.416 & 0.79 & $(0.52-1.1)$ & 0.271
\end{tabular}

IC 95\%. intervalo de confianza del 95\%; OR: odds ratio.

*Estadísticamente significativo.

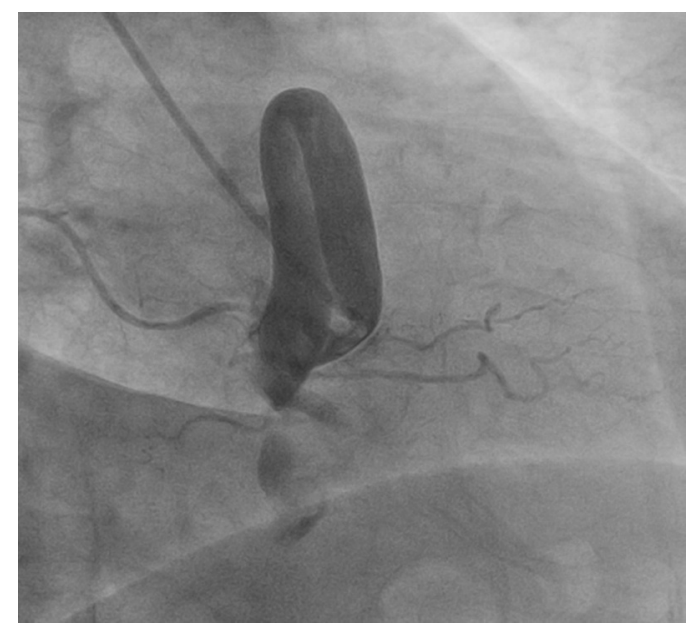

Figura 1. Ectasia en arteria coronaria derecha. Imagen que muestra coronaria derecha ectásica, con imagen de trombo en segmento vertical y flujo TIMI 0 en un paciente con ectasia coronaria Markis I.

coronaria, lo que corresponde a una relación 12:1, lo que concuerda con resultados obtenidos en otros estudios retrospectivos ${ }^{1,4}$.

En nuestra investigación la enfermedad comprometió más de dos vasos en más de la mitad de los casos, lo que difiere del estudio realizado por Zhang ${ }^{5}$, en donde el $72.4 \%$ de los pacientes con ectasia coronaria tenían afectación de un solo vaso (Markis tipo III y IV).

Investigaciones previas han evaluado la presencia de ectasia coronaria desde otra perspectiva, ya sea su incidencia, pronóstico, mortalidad o coexistencia de enfermedad arterial coronaria, sin embargo ninguno de ellos se enfoca en pacientes con infarto de miocardio ${ }^{5-7}$.
Como factores de riesgo asociados a la ectasia coronaria encontramos la presencia de diabetes, sobrepeso y obesidad y como factores de riesgo independiente hipercolesterolemia y la exposición a herbicidas. Similar a nuestros resultados, el estudio de Armirzadegan, et al. ${ }^{8}$, realizado en la población de Irán, encontró que el sobrepeso y obesidad fueron más frecuentes y con diferencia significativa en pacientes con ectasia coronaria en comparación con pacientes con estenosis coronaria.

La hipercolesterolemia como factor de riesgo independiente en la ectasia coronaria tiene resultados variables, en relación con la población analizada. En un estudio realizado en pacientes de Suecia e Irlanda por Boles, et al. ${ }^{11}$, si bien se encontró con mayor frecuencia hiperlipidemia en pacientes con ectasia coronaria, esta no fue significativa; en nuestro estudio encontramos asociación con hipercolesterolemia.

Encontramos una prevalencia de ectasia coronaria del $8.5 \%$, siendo de las más altas documentadas en el mundo, en el contexto de pacientes con infarto agudo de miocardio a los que se les efectuó coronariografía. Esta prevalencia es superior a lo descrito en Arabia Saudita del 6\%, el cual ha reportado las prevalencias más elevadas, no obstante, solo el $57 \%$ de ellos contaban con antecedente de infarto de miocardio ${ }^{12}$.

Se encontraron diferencias hematológicas entre ambos grupos como en el caso de la hemoglobina en personas con ectasia, que fue ligeramente mayor en comparación con aquellos sin ectasia. El IPL es un marcador pronóstico y diagnóstico en la enfermedad arterial coronaria, sus niveles elevados se asocian con pronóstico desfavorable en pacientes con síndrome 
coronario agudo al revelar cambios en los recuentos de plaquetas y linfocitos debido a estados inflamatorios y protrombóticos agudos. Solo existe un estudio realizado en este sentido en el contexto de infarto agudo de miocardio y ectasia coronaria, en Turquía por Kundi, et al..$^{15}$, que encontraron que los pacientes con ectasia coronaria tuvieron un IPL significativamente mayor y se consideró como un factor de riesgo comparado con pacientes con enfermedad coronaria obstructiva. El valor elevado del IPL también se encontró en nuestro estudio, sin embargo no se midió en forma longitudinal.

El ancho de distribución eritrocitario refleja la variabilidad en el tamaño de los glóbulos rojos circulantes (anisocitosis) y se ha asociado a eventos cardiovasculares por mecanismos inflamatorios y activación neurohumoral, teniendo un rol patogénico en la enfermedad coronaria aterosclerosa. En un estudio realizado por Dogdu ${ }^{16}$ en donde evaluaron un grupo de pacientes con ectasia coronaria y otro grupo control sin ectasia encontraron que los niveles de ancho de distribución eritrocitario (ADE) eran significativamente más elevados en el grupo con ectasia coronaria en comparación con el control. Por el contrario, en nuestro estudio se encontró un nivel de ADE ligeramente mayor en el grupo sin ectasia coronaria.

Finalmente, la exposición a herbicidas presentó un mayor peso sobre la ectasia acompañando al infarto. Los componentes más comunes a los herbicidas son 2,4,5-T (ácido tricloropenoxiacético), 2,4 D (ácido diclorofenoxiacético) o un inhibidor de colinesterasa. La exposición crónica a estos agentes puede elevar los niveles de acetilcolina en una región focal del intersticio coronario por medio de inhibición competitiva, incrementando los productos finales de acetilcolina, estos son estimuladores potentes de óxido nítrico, factor de relajación derivado de endotelio ${ }^{17}$. En México, al igual que en otros países, el uso de herbicidas continúa a pesar de los efectos deletéreos sobre el ambiente y la toxicidad, incluyendo su asociación con ectasia coronaria.

Deben tenerse en cuenta algunas limitaciones del estudio, debido a su diseño transversal no se dio seguimiento a los pacientes. El IPL solo se midió una vez, por lo que desconocemos su valor pronóstico. Otra limitación es el sesgo de referencia de tercer nivel, no se precisó el tipo de herbicida, vía y tiempo de exposición, además de no evaluarse otros factores de riesgo tales como la hiperhomocisteinemia, entre otros factores ${ }^{18}$.
Consideramos que los resultados de este estudio son relevantes debido a la alta prevalencia de ectasia coronaria que se encontró en esta población con infarto de miocardio. Un estudio reciente realizado en China encontró que de 11,267 angiografías efectuadas en pacientes con sospecha de enfermedad arterial coronaria únicamente el $1 \%$ correspondían a ectasia coronaria ${ }^{19}$.

\section{Conclusiones}

Se encontró una elevada prevalencia de ectasia coronaria en pacientes con infarto de miocardio, siendo los principales factores de riesgo la hipercolesterolemia y exposición a herbicidas. El IPL se encontró elevado en pacientes con ectasia coronaria, siendo un reflejo del proceso inflamatorio. La detección oportuna de esta entidad es importante debido a su asociación con eventos coronarios agudos. Se requieren estudios prospectivos que valoren el pronóstico de estos pacientes a largo plazo.

\section{Financiamiento}

Para la realización del presente estudio no se requirió de financiamiento externo.

\section{Conflicto de intereses}

Ninguno de los autores presenta conflicto de intereses.

\section{Responsabilidades éticas}

Protección de personas y animales. Los autores declaran que para esta investigación no se han realizado experimentos en seres humanos ni en animales.

Confidencialidad de los datos. Los autores declaran que han seguido los protocolos de su centro de trabajo sobre la publicación de datos de pacientes.

Derecho a la privacidad y consentimiento informado. Los autores han obtenido el consentimiento informado de los pacientes y/o sujetos referidos en el artículo. Este documento obra en poder del autor de correspondencia.

\section{Bibliografía}

1. Valente S, Lazzeri C, Giglioli C, Sani F, Romano SM, Margheri M, et al. Clinical expression of coronary artery ectasia. J Cardiovasc Med. 2007:8(10):815-20.

2. Boles U, Zhao Y, Rakhit R, Shiu MF, Papachristidis A, David S, et al. Patterns of coronary artery ectasia and short term outcome in acute myocardial infarction. Scand Cardiovasc J. 2014;48(3):161-6. 
3. Ozgur UO, Sadi G. Coronary artery ectasia. Cor Vasa. 2013;55(3):242-47.

4. EIGuindy MS, EIGuindy AM. Aneurysmal coronary artery disease: an overview. Glob Cardiol Sci Pract. 2017;2017(3):1-17.

5. Zhang Y, Huang Q-J, Li X-L, Guo Y-L, Zhu C-G, Wang X-W, et al. Prognostic value of coronary artery stenoses, Markis class, and ectasia ratio in patients with coronary artery ectasia. Cardiology. 2015; 131(4):251-59.

6. Lam CSP, Ho KT. Coronary artery ectasia: a ten year experience in a tertiary hospital in Singapore. Ann Acad Med Singapore. 2004; 33(4):419-22.

7. Nyamu P, Ajit MS, Joseph PK, Venkitachalam L, Sugirtham NA. The prevalence and clinical profile of angiographic coronary ectasia. Asian Cardiovasc Thorac Ann. 2003;56(5):122-26.

8. Armirzadegan AR, Davoodi G, Soleimani A, Lotfi M, Hakki E, Shabpiray $\mathrm{H}$, et al. Association between traditional risk factors and coronary artery ectasia: a study on 10,057 angiographic procedures among Iranian population. J Tehran Heart Cent. 2014;9(1):27-32.

9. Yilmaz H, Sayar N, Yilmaz M, Tangürek B, Cakmak N, Gürkan U, et al. Coronary artery ectasia: clinical and angiographical evaluation. Arch Turk Soc Cardiol. 2009;36(8):530-35.

10. Pinar E, López R, Lozano I, Cortés R, Carrillo P, Rodríguez R, et al Coronary ectasia: prevalence, and clinical and angiographic characteristics. Rev Esp Cardiol. 2003;56(5):473-79.
11. Boles U, Zhao Y, David S, Eriksson P, Henein MY. Pure coronary ectasia differs from atherosclerosis: morphological and risk factors analysis. Int J Cardiol. 2012;155(2):321-23.

12. Almansori MA, Elsayed HA. Coronary artery ectasia - A sample from Saudi Arabia. J Saudi Heart Assoc. 2015;27(3):160-63.

13. Palomo VJA, Necoechea JC, Ruesga E, Díaz E, Muñoz ME, Jáuregui R, et al. Ectasia de las arterias coronarias. Prevalencia y significancia clínica. Rev Mex Cardiol. 1997;8(1):15-20.

14. Ruiz-Morales JM, García-López SM, González-Chon O. Síndromes coronarios agudos como forma presentación clínica de arterias coronarias ectásicas. Rev Invest Med Sur Mex. 2012;19(3):140-43.

15. Kundi H, Gök M, Çetin M, Kızıltunç E, Çiçekcioğlu H, Güven Z, et al. Relationship between platelet-to-lymphocyte ratio and the presence and severity of coronary artery ectasia. Anatol J Cardiol. 2016;16(11):857-62.

16. Dogdu O, Fatit K, Nihat K, Mikail Y, Deniz E, Metin K, et al. Assessment of red cell distribution width (RDW) in patients with coronary artery ectasia. Clin Appl Thromb Hemost. 2012;18(2):211-4.

17. England JF. Herbicides and coronary artery ectasia. Med J Aust. 1981;1(3):140.

18. Devabhaktuni S, Mercedes A, Diep J, Ahsan C. Coronary artery ectasia-A review of current literature. Curr Cardiol Rev. 2016;12(4):318-23.

19. Jiang X, Zhou P, Wen C, Yin Z, Liu T, Xu M, et al. Coronary anomalies in 11,267 southwest chinese patients determined by angiography. Biomed Res Int. 2021;2021:6693784. 